\title{
On primitive recursive real numbers *
}

\author{
by \\ R. S. Lehman (Berkeley, Cal.)
}

The concept of primitive recursiveness can be used to give constructive versions of various classical definitions of real numbers. The class $\Re_{1}$ determined by the analogue of Cantor's definition in terms of Cauchy sequences was called by Specker [9] the class of primitive recursive real numbers. Specker also considered the class $\mathfrak{R}_{2}$ of real numbers with primitive recursive decimal expansions and the class $\Re_{3}$ corresponding to the Dedekind cut definition of real numbers. He proved that $\Re_{\mathbf{B}} \subset \Re_{2}$ $\subset \Re_{1}$, where the inclusions are proper; and he obtained other results concerning numbers in these classes. Further results have been obtained by Péter [6], [7] and Mostowskiki [4].

The questions discussed in the present paper have as a common feature the property that either the questions themselves or the methods used here to study them are concerned with the regular continued fraction expansions of real numbers. The present study began with an attempt to determine whether the seemingly lawless expansion for $\pi$ is primitive recursive. (1) (See [5], p. 35, [3].) An answer to this question is furnished by Theorem 1 , which asserts that the class of irrational numbers with primitive recursive continued fractions is identical with the class of primitive recursive real numbers which are recursively irrational in the sense of Péter [6]. Since it is known that $\pi$ is recursively irrational (see Goodstein [1]), it follows that its continued fraction expansion is primitive recursive.

In Section 3 we use some results of Hurwitz [2], concerning Farey series and continued fraction expansions, to give an example of an irrational number in $\Re_{3}$ which is not recursively irrational.

Mostowski [1] has asked whether the class $\Re_{3}$ is identical with the class of numbers with primitive recursive $b$-adic expansions for every integer $b>1$. In Section 4 we use a result from analytic number theory

* Part of the research for this paper was done undex a Fulbright grant for research at the University of Göttingen.

(2) The author is indebted to Professor Saul Gorn for the suggestion that the rague question of whether there is a law for the continued fraction for $\pi$ might have a meaningful version in terms of recursive functions. 
concerning the distribution of primes in arithmetic progressions to obtain a negative answer to this question.

If one uses the concept of general recursiveness instead of that of primitive recursiveness, then the definitions corresponding to those mentioned above turn out to be equivalent. Mostowslki [4] has, however, shown that sifnilar distinctions then arise when one considers sequences instead of numbers. In the present paper we shall be exclusively concerned with primitive recursiveness; therefore, to shorten statements, instead of saying "primitice recursive" we shall often say simply "recursive"

\$1. Throughout; this paper we shall limit our considerations to positive rational numbers and positive real numbers. An extension to negative numbers; would offer no real difficulty but would complicate the exposition somewhat. First, let us summarize the portion of the elementary theory of continued fractions which we sluall use. For proofs we refer to Chapters 1 and 2 of [5].

Every positive irrational number $a$ has a unique infinite regular continued fraction expansion of the form

$$
\alpha=b_{0}+\frac{1}{b_{1}+\frac{1}{b_{2}+\ldots}}
$$

where the $b_{n}$ are integers, $b_{0} \geqslant 0$ and $b_{n} \geqslant 1 \quad(n=1,2,3, \ldots)$. As is customary, for the sake of brevity, we write $a=\left[b_{0}, b_{1}, b, \ldots\right]$. The number $b_{n}$ is called the partial quotient of order $n$.

We consider the sequences $p_{-1}, p_{0}, p_{1}, p_{2}, \ldots$ and $q_{-1}, q_{0}, q_{1}, q_{2}, \ldots$ determined by the recurrence relations

$$
\begin{aligned}
& \because \quad p_{-1}=1, \quad q_{-1}=0 \text {, } \\
& \text { (1.1) } p_{0}=b_{0}, \quad q_{0}=1 \text {, } \\
& \therefore p_{n+1}=b_{n+1} p_{n}+p_{n-1}, \quad q_{n+1}=b_{n+1} q_{n}+q_{n-1} \quad(n=0,1,2, \ldots) \text {. }
\end{aligned}
$$

The fraction $p_{n} / q_{n}$ is called the convergent of order $n$. Because of the relation :

$(1,2)$

$$
p_{n} q_{n-1}-q_{n} p_{n-1}=(-1)^{n-1} \quad(n=0,1,2, \ldots),
$$

these fractions are reduced fractions. The sequence $\left\{p_{n} / q_{n}\right\}$ converges to the number $\alpha$. In fact

$$
\begin{array}{ll}
(1.3) & \frac{1}{q_{n}\left(q_{n+1}+q_{n}\right)}<\left|\alpha-\frac{p_{n}}{q_{n}}\right|<\frac{1}{q_{n} q_{n+1}} \quad(n=0,1,2, \ldots) . \\
\therefore 110 & \frac{p_{0}}{q_{0}}<\frac{p_{2}}{q_{2}}<\frac{p_{4}}{q_{4}}<\ldots<\alpha<\ldots<\frac{p_{5}}{q_{5}}<\frac{p_{3}}{q_{3}}<\frac{p_{1}}{q_{1}} .
\end{array}
$$

We shall use the following formula which together with (1.1) gives a recursion scheme for computing the partial quotients $\left({ }^{2}\right)$ :

$$
b_{n+1}=\left[\frac{p_{n-1}-\alpha q_{n-1}}{\alpha q_{n}-p_{n}}\right] \quad(n=0,1,2, \ldots) \text {. }
$$

The continued fraction convergents provide best approximations to $\alpha$ in the following sense. If $n>1,0<q \leqslant q_{n}$, and $p / q \neq p_{n} / q_{n}$, then

$$
\left|\alpha-\frac{p_{n}}{q_{n}}\right|<\left|a-\frac{p}{q}\right| \text {. }
$$

Also if $p$ and $q$ are positive integers and

$$
\left|\alpha-\frac{p}{q}\right|<\frac{1}{2 q^{2}}
$$

then $p / q$ is equal to a convergent to $a$.

\$2. A sequence of rational numbers $\left\{a_{n}\right\}$ is said to be recursive if and only if there are recursive functions $\varphi(n)$ and $\psi(n)$ such that $\psi(n) \geqslant 1$ and $a_{n}=\varphi(n) / \psi(n)$. The sequence $\left\{a_{n}\right\}$ is said to converge recursively if and only if there is a recursive function $\tau(m)$ such that

$$
\left|a_{n}-a_{n *}\right|<\frac{1}{m} \quad \text { for } \quad n, n^{*} \geqslant \tau(m) \text {. }
$$

A real number $r$ is said to be a recursive real number if and only if there is a recursive sequence of rational numbers which converges recursively to $r$.

Péter [6], [7] has introduced the concept of a recursive real number being recursively irrational. We shall use a definition which is easily proved to be equivalent to hers. We say that a real number $\alpha$ is recursively irrational if and only if there is a recursive function $v(n)$ such that for all positive integers $m$ and $n$

$$
\left|\alpha-\frac{m}{n}\right|>\frac{1}{v(n)}
$$

We say that the continued fraction expansion $\alpha=\left[b_{0}, b_{1}, b_{2}, \ldots\right]$ is recursive if and only if $b_{n}$ is a recursive function of $n$. Our first result is the following theorem.

THEOREM 1. An irrational number a has a primitive recursive continued fraction expansion if and only if it is a recursive real number which is recursively irrational.

(2) By $[x]$ we shall always mean the greatest integer which is less than or equal to $x$. 
Proof. First we assume that $a$ is a recursive real number which is recursively irrational and prove that its continued fraction expansion is recursive. By assumption there is then a recursive function $v(n)$ such that (2.1) holds for $m, n \geqslant 1$. We shall find it convenient to replace $v(n)$ by a recursive function $\nu^{*}(n)$ defined by the recursion

$$
\begin{aligned}
\nu^{*}(0) & =\nu(0), \\
\nu^{*}(n+1) & =\max \left(\nu(n+1), \nu^{*}(n)\right) .
\end{aligned}
$$

It is then easy to establish that $\nu^{*}(n)$ is a monotone increasing function and that (2.1) holds with $\nu$ replaced by $\nu^{*}$.

Next we define a recursive function $\mu(n)$ by the equation

$$
\begin{aligned}
\mu(0) & =1 \\
\mu(n+1) & =\nu^{*}((\mu(n)) .
\end{aligned}
$$

We can then prove that if $p_{n} / q_{n}$ is the convergent of order $n$ to $\alpha$, then

$$
q_{n} \leqslant \mu(n) \quad(n=0,1,2, \ldots) .
$$

Indeed, by (2.1) and (1.3) we have

$$
\frac{1}{v^{*}\left(q_{n}\right)}<\left|a-\frac{p_{n}}{q_{n}}\right|<\frac{1}{q_{n} q_{n+1}} .
$$

Hence, since $q_{n} \geqslant 1$ for $n \geqslant 0, q_{n+1}<\nu^{*}\left(q_{n}\right)$. Because $q_{0}=1=\mu(0)$ and because $\nu^{*}$ is monotone, the inequality (2.2) follows by induction.

Since $a$ is a recursive real number, there are recursive functions $\varphi(k), \psi(k)$, and $\tau(m)$ such that $\psi(k) \geqslant 1$,

and

$$
\frac{\varphi(k)}{\psi(k)} \rightarrow \alpha \quad \text { as } \quad k \rightarrow \infty,
$$

$$
\left|\frac{\varphi(k)}{\psi(k)}-\frac{\varphi\left(k^{*}\right)}{\psi\left(k^{*}\right)}\right|<\frac{1}{m} \quad \text { for } \quad k, k^{*} \geqslant \tau(m) .
$$

Letting $k^{*} \rightarrow \infty$, we find that

$$
\left|\frac{\varphi(k)}{\varphi(k)}-\alpha\right| \leqslant \frac{1}{m} \quad \text { for } \quad k \geqslant \tau(m) .
$$

Let $a=\left[b_{0}, b_{1}, b_{2}, \ldots\right]$ and let $p_{n} / q_{n}$ and $p_{n+1} / q_{n+1}$ be the convergents to $a$ of orders $n$ and $n+1$. Then if $\beta$ is any real number for which

$$
|\alpha-\beta|<\left|\alpha-\frac{p_{n+1}}{g_{n+1}}\right|,
$$

the partial quotients of order $\leqslant n$ are the same in the expansions of $\alpha$ and $\beta$. To see this, we observe that $\alpha$ lies between $p_{n} / q_{n}$ and $p_{n+1} / q_{n+1}$.
Also $\alpha$ is closer to $p_{n+1} / q_{n+1}$ than it is to $p_{n} / q_{n}$. Hence $\beta$ must lie between $p_{n} / q_{n}$ and $p_{n+1} / q_{n+1}$, and since these two numbers have the same partial quotients of order $\leqslant n$, so must $a$ and $\beta$. (See [5], p. 35.)

We apply this result with

$$
\beta=\frac{\varphi(\omega(n))}{\psi(\omega(n))}, \quad \omega(n)=\tau\left(\nu^{*}(\mu(n+1))\right) .
$$

The assumptions are satisfied since by $(2.1),(2.2)$, and (2.3)

$$
\left|\alpha-\frac{p_{n+1}}{q_{n+1}}\right|>\frac{1}{y^{*}\left(q_{n+1}\right)} \geqslant \frac{1}{y^{*}(\mu(n+1))} \geqslant\left|\alpha-\frac{\varphi(\omega(n))}{\psi(\omega(n))}\right|=|\alpha-\beta| .
$$

By (1.5) and (1.4) we have

$$
\begin{array}{rlrl}
b_{0} & =\left[\frac{\varphi(\omega(0))}{\psi(\omega(0))}\right], & b_{1} & =\left[\frac{\psi(\omega(1))}{\mid b_{0} \psi(\omega(1))-\varphi(\omega(1))}\right], \\
p_{0}=b_{0}, & p_{1}=b_{1} p_{0}+1 \\
q_{0}=1, & q_{1}=b_{1} q_{0},
\end{array}
$$

$$
\begin{aligned}
& b_{n+1}=\left[\frac{\mid p_{n-1} \psi(\omega(n+1))-q_{n-1} \varphi(\omega(n+1)||}{\left|q_{n} \varphi(\omega(n+1))-p_{n} \psi(\omega(n+1))\right|}\right] \quad(n=1,2,3, \ldots), \\
& p_{n+1}=b_{n+1} p_{n}+p_{n-1} \quad(n=1,2,3, \ldots), \\
& q_{n+1}=b_{n+1} q_{n}+q_{n-1} \quad(n=1,2,3, \ldots) .
\end{aligned}
$$

The functions $[a / b],|a-b|, \varphi, \psi$, and $\omega$ are all primitive recursive. The scheme (2.4) is a combination of a simultaneous recursion with a course of values recursion. Such recursions can always be reduced to primitive recursions (see Péter [7], pp. 32-36). Consequently $b_{n}, p_{n}$, and $q_{n}$ are recursive functions of $n$. This completes the proof that a recursively irrational recursive real number has a recursive continued fraction expansion.

Next, we assume that $\alpha$ has a recursive continued fraction expansion and show that it is a recursive real number which is recursively irrational. Since $b_{n}$ is a recursive function, so also are $p_{n}$ and $q_{n}$ defined by the relations (1.1). We know that the sequence $\left\{p_{n} / q_{n}\right\}$ converges to $\alpha$. Moreover, it converges recursively; for if $n, n^{*}>m$, we have by (1.4) and (1.2)

$$
\left|\frac{p_{n}}{q_{n}}-\frac{p_{n^{*}}}{q_{n^{*}}}\right| \leqslant\left|\frac{p_{m}}{q_{m}}-\frac{p_{m+1}}{q_{m+1}}\right|=\frac{\left|p_{m} q_{m+1}-p_{m+1} q_{m}\right|}{q_{m} q_{m+1}}=\frac{1}{q_{m} q_{m+1}}<\frac{1}{m} .
$$

Hence by letting $\tau(m)=m$ we see that $a$ is a recursive real number. 
Next we prove that $\alpha$ is recursively irrational. By (1.6) and (1.3) we know that for all integers $p$ and all positive integers $q \leqslant q_{n}$

$$
\left|\alpha-\frac{p}{q}\right| \geqslant\left|\alpha-\frac{p_{n}}{q_{n}}\right|>\frac{1}{q_{n}\left(q_{n}+q_{n+1}\right)} .
$$

In particular, since $n \leqslant q_{n}$, we have

If we take

$$
\begin{gathered}
\left|a-\frac{m}{n}\right|>\frac{1}{q_{n}\left(q_{n}+q_{n+1}\right)} \quad \text { for } \quad m, n>0 . \\
\nu(n)=q_{n}\left(q_{n}+q_{n+1}\right) \quad(n=0,1,2, \ldots)
\end{gathered}
$$

then (2.1) will be satisfied. Consequentily $\alpha$ is recursively irrational.

\$3. A real number $r$ is said to determine a recursive cut if and only if the relation $m /(n+1)<r$ is recursive (see Specker [9]). Péter [6] has proved that the number $\alpha$ determines a recursive cut if and only if [ $n \alpha]$ is recursive.

Péter [6] has shown that if a recursive real number $\alpha$ is recursively irrational then the sequence $[n \alpha]$ is recursive. Let us give a short proof of this fact based on the result that such a number has a recursive continued fraction expansion. Since $q_{2 n+1} \geqslant n$, by (1.3) and (1.4) we have

$$
0<n \alpha-\frac{n p_{2 n}}{q_{2 n}}<\frac{n}{q_{2 n} q_{2 n+1}} \leqslant \frac{1}{q_{2 n}} .
$$

$\Delta t$ least $1 / q_{2 n}$ must be added to $n p_{2 n} / q_{2 n}$ to change its integral part. Hence,

$$
[n \alpha]=\left[n p_{2 n} / q_{2 n}\right] \text {. }
$$

If the continued fraction expansion is recursive, then $p_{n}$ and $q_{n}$ are recursive; and it then follows that [na] is recursive.

This result raises the question of whether there are irrational numbers $\alpha$ which determine recursive cuts but are not recursively irrational. To answer this question we shall use some results of Hurwitz concerning the connection between Farey series and regular continued fractions.

For this section we shall limit the discussion to irrational numbers for which $0<a<1$. The Farey series of order $n$ is a listing in order of magnitude of all reduced fractions $p / q$ for which $0 \leqslant p \leqslant q$ and $0<q \leqslant n$. Two fractions $r / s$ and $u / v$ which are adjacent in the Farey series of order $n$ remain as adjacent fractions in Farey series of larger order until the order increases to $s+v$. Then in the series of order $s+v$ the fraction $(r+u) /(s+v)$ is inserted between $r / s$ and $u / v$.

We define a function $\chi(n)$ which we call the Hurwitz characteristic of $\alpha$. (See [2].) Let $\chi(0)=0$. The Farey series of order 1 is $0 / 1,1 / 1$. The Farey series of order 2 is obtained by inserting the fraction $1 / 2$ between $0 / 1$ and $1 / 1$. If $a<1 / 2$, then we let $\chi(1)=0$; if $a>1 / 2$, we let $\chi(1)=1$. In general, after $n-1$ steps $\chi(n-1)$ has been determined and $\alpha$.has been located between two adjacent fractions in some Farey series, say $r / s$ and $u / v$. Then if $a<(r+u) /(s+v)$, we let $\chi(n)=0$; and if this is not the case, we let $\chi(n)=1$. Observe that if the characteristic is known, it is easy to determine the position of $\alpha$ with respect to each of the Farey series.

From the characteristic of $\alpha$ it is also very easy to find the regular, continued fraction expansion of $a$. Suppose that the first $b_{1}$ values in the sequence $\{x(n)\}$ are 0 , the next $b_{2}$ values are 1 , the next $b_{3}$ values are 0 , etc., where $b_{i} \geqslant 1 \quad(i=1,2,3, \ldots)$. Then Hurwitz proved that $a=\left[0, b_{1}, b_{2}, \ldots\right]$.

We prove the following theorem which gives a new characterization of the set of numbers which determine recursive cuts.

THEoren 2. An irrational number a belween 0 and 1 determines. a recursive cut if and only if its Humvitz characteristic $\chi(n)$ is recursive.

Proof. First we describe formally the construction of $\chi(n)$ for a given irrational number $\alpha$. Let

$$
\begin{array}{lll}
\varrho(m, n)=0 & \text { if } & \alpha<m /(n+1), \\
\varrho(m, n)=1 & \text { if } & \alpha>m /(n+1) .
\end{array}
$$

Let $\left(\frac{v_{1}(n)}{i_{2}(n)}, \frac{v_{3}(n)}{v_{4}(n)}\right)$ be the interval in which $a$ has been located after the nth step of the construction. Then we have

$$
\begin{gathered}
z(0)=0, \quad v_{1}(0)=0, \quad v_{2}(0)=v_{3}(0)=v_{4}(0)=1 \\
\left.\chi(n+1)=\varrho\left(v_{1}(n)+\nu_{3}(n), \quad v_{2}(n)+v_{4}(n)-1\right)\right)
\end{gathered}
$$

$$
\begin{aligned}
& \nu_{1}(n+1)=\nu_{1}(n)+\chi(n+1) \nu_{3}(n), \\
& v_{2}(n+1)=\nu_{2}(n)+\chi(n+1) v_{4}(n), \\
& v_{3}(n+1)=\nu_{3}(n)+(1-\chi(n+1)) \nu_{1}(n), \\
& \nu_{4}(n+1)=\nu_{4}(n)+(1-\chi(n+1)) \nu_{2}(n) .
\end{aligned}
$$

If $\alpha$ determines a recursive cut, then the function $\varrho(m, n)$ is recursive. The equations (3.1) and (3.2) then define $\chi(n), v_{1}(n), v_{2}(n), v_{3}(n)$, and $v_{4}(n)$ simultaneously as recursive functions,

On the other hand, if $\alpha$ is an irrational number which has a recursive function $\chi(n)$ as its characteristic, then the equations (3.2) define $v_{1}(n)$, $v_{2}(n), v_{3}(n)$ and $v_{4}(n)$ as recursive functions. One can prove by induction that $\max \left(v_{2}(n), v_{4}(n)\right)>n$. Consequently $\nu_{1}(n) / v_{2}(n)$ and $\nu_{3}(n) / v_{4}(n)$ are 
adjacent fractions in some Farey series of order greater than $n$. It follows that there can be no number $m / n$ for which

$$
\frac{\nu_{1}(n)}{v_{3}(n)}<\frac{m}{n}<\frac{\nu_{3}(n)}{\nu_{4}(n)}
$$

Hence $[n a]=\left[n v_{1}(n) / v_{2}(n)\right]$, and it then follows that $[n a]$ is recursive. This establishes that $\alpha$ determines a recursive cut.

The following lemma, which is essentially Specker's Lemma II, will be used in the proofs of Theorems 3 and 4 . By " $\mu_{x}$ " we shall mean "the smallest natural number $x$ such that ...". We shall use this operator only when there is an $x$ for which the condition holds.

LEMMA 1. There is a primitive recursive function $\lambda(n)$ which takes no values except 0,1 , and 2 , suoh that for every $n$ there is an $x \geqslant n$ such that $\lambda(x) \neq 2$ but the function

$$
\lambda\left(\mu_{x}(x \geqslant n \& \lambda(x) \neq 2)\right)
$$

is not a primitive reeursive function of $n$.

Proof. Specker's Lemma II asserts that there is a primitive recursive predicate $\mathfrak{A}(n)$ and a recursive function $\gamma(n)$, taking only the values 0 and 1 , such that for every $n$ there is an $x \geqslant n$ for which $\mathfrak{U}(x)$ holds but

$$
\gamma\left(\mu_{x}(x \geqslant n \& \mathfrak{N}(x))\right)
$$

is not recursive. We let $\lambda(n)=2$ if $\mathfrak{X}(n)$ does not hold, and we let $\lambda(n)$ $=\gamma(n)$ if $\mathscr{U}(n)$ does hold. Lemma 1 then follows immediately.

Observe also that the function

$$
\sigma(n)=\mu_{x}(x \geqslant n \& \lambda(x) \neq 2)
$$

cannot be primitive recursive.

THEOREM 3. There is an irrational number a which determines a recursive cut but is not recursively irrational.

Proof. We use Lemma 1 to define an appropriate Hurwitz characteristic $\chi(n)$. We let $\chi(0)=0$. In general, if $\lambda(n)=2$ we let $\chi(n+1)=\chi(n)$; if $\lambda(n) \neq 2$ we let $\chi(n+1)=1-\chi(n)$. The function $\chi(n)$ so defined is recursive and hence by Theorem 2 defines a number $a$ which determines a recursive cut. Let

$$
\sigma(n)=\mu_{x}(x \geqslant n \& \lambda(x) \neq 2), \quad .
$$

and let $a=\left[0, b_{1}, b_{2}, b_{3}, \ldots\right]$. According to Hurwitz's results the sequence $\{\chi(n)\}$ consists of $b_{1} 0$ 's followed by $b_{2} 1$ 's, etc. Consequently

$$
\sigma(n)=\mu_{x}\left(x \leqslant \sum_{i=1}^{n+1} b_{i} \& x \geqslant n \& \lambda(x) \neq 2\right) .
$$

If $a$ were recursively irrational, it would have a recursive continued fraction expansion; and the function $\sum_{i=1}^{n+1} b_{i}$ would thus be a recursive function of $n$. It would then follow that $\sigma(n)$ is recursive, contrary to Lemma 1 . Hence we conclude that $\alpha$ is not recursively irrational.

8. Let $b$ be an integer $\geqslant 2$. A real number $r$ is said to have a recursive $b$-adic expansion if and only if there is a primitive recursive function $\gamma(n)$ such that

$$
r=\sum_{n=0}^{\infty} \gamma(n) b^{-n}
$$

and $0 \leqslant \gamma(n)<b$ for $n=1,2,3, \ldots$

The following theorem answers a question of Mostowski [4].

THEOREM 4. There is an irrational number a whioh has a recursive $b$-adic expansion for all $b \geqslant 2$ but does not determine a recursive cut.

In the proof of Theorem 4 we shall use the following lemma concerning primes in arithmetic progressions.

LEMMA 2. If $l$ is relatively prime to $k$ and if $n>0$; let $p(n, k, l)$ be the $n$-th prime which is congruent to $l$ modulo $k$. In all other cases let $p(n, k, l)$ $=0$. Then the function $p(n, k, l)$ is primitive recursive.

Proof. The function $p(n, k, l)$ is well defined by Dirichlet's theorem. Let $\pi(x, k, l)$ be the number of primes $p$ for which $p \leqslant x$ and $p \equiv l(\bmod k)$. It is known (see [8], p. 144) that there is a positive number $O$ such that if $l$ is relatively prime to $k$, and $1 \leqslant k \leqslant \log x$ then

$$
\pi(x, k, l)>\frac{x}{2 k \log x}-C
$$

We may assume $C>3$. Taking $x=e^{k(n+C)}$, we find

$$
\pi\left(e^{k(n+C)}, k, l\right)>\frac{(n+C) e^{k(n+C)}}{2 k^{2}(n+C)^{2}}-C>n
$$

since $e^{u} / 2 u^{2}>1$ when $u>3$. Thus in a search to find the $n$th prime $p$ for which $p \equiv l(\bmod k)$ one need not search further than $e^{k(n+C)}$. This yields a primitive recursive bound for use with the $\mu$ operator in defining $p(n, k, l)$ as a primitive recursive function. The completion of the formal definition offers no difficulties.

Proof of Theorem 4. Since this proof is somewhat complicated, we first give a rough outline of the proof. Using the function $\lambda(n)$ of Lemma 1 we generate a recursive sequence of rational numbers $\{\varphi(n) / \psi(n)\}$ which converges to a number $a$. This sequence is chosen so that for long stretches the same fraction appears repeatedly. This is done 
in such a way that the relation $\alpha<\varphi(n) / \psi(n)$ is not recursive and so that the numbers $\psi(n)$ are all primes. Moreover, the sequence is chosen so that all rational numbers which are "good approximations" to $\alpha$ are in the sequence. Roughly speaking, one can say that the only way a recursive real number can fail to have a recursive $b$-adic expansion is by being approximated too well by an infinite sequence of fractions of the form $a / b^{n}$. But since all good approximations to $a$ have denominators which are primes, no such sequence can exist; and $\alpha$ must, therefore, have a recursive $b$-adic expansion.

For the exact proof we let $\lambda(n)$ be the recursive function described by Lemma 1 and we let $p(n, k, \eta)$ be the recursive function of Lemma 2 . We define simultaneously the functions $\varphi(n), \psi(n), \xi(n), \eta(n), \varkappa(n)$, and $\varrho(n)$ as primitive recursive functions by the equations

$$
\varphi(0)=1, \psi(0)=2, \xi(0)=0, \eta(0)=1, \varrho(0)=0, x(0)=0,
$$

$$
\left.\begin{array}{ll}
\varphi(n+1)=\varphi(n), & \psi(n+1)=\psi(n) \\
\xi(n+1)=\xi(n), & \eta(n+1)=\eta(n), \\
\varrho(n+1)=\varrho(n), & x(n+1)=x(n)+1
\end{array}\right\} \quad \text { if } \quad \lambda(n)=2,
$$$$
\varrho(n+1)=\lambda(n) ; \quad x(n+1)=0
$$

$$
\left.\xi(n+1)=\varphi(n), \quad \eta(n+1)=\psi(n)^{2}\right\} \quad \text { if } \quad \lambda(n)=0 \text { or } 1
$$

$\psi(n+1)=p(x(n)+3, \psi(n), \eta(n))$

$\varphi(n+1)=[\psi(n+1) / \psi(n)] \cdot \varphi(n)+\xi(n)\}$

if $\quad \lambda(n)=1-\varrho(n)$,

$\because \quad \psi(n+1)=p(x(n)+3, \psi(n), \psi(n)-\eta(n))$

$\varphi(n+1)=[\psi(n+1) / \psi(n)] \cdot \varphi(n)+\varphi(n)-\xi(n)$

if $\lambda(n)=\varrho(n)$.

The numbers $\varphi(n) / \psi(n)$ form a sequence which we shall prove converges to an irrational $a$. The number $\xi(n) / \eta(n)$ is the last member of the sequence which precedes the number $\varphi(n) / \psi(n)$ and differs from it; $\varrho(n)$ is 0 or 1 depending on whether $\xi(n) / \eta(n)$ is less or greater than $\alpha$; and $x(n)$ is a counter which keeps a record of how many earlier members of the sequence are equal to $\varphi(n) / \psi(n)$.

We define a function $f(n)$ by the equations

(4.2) $f(0)=-1, \quad f(m+1)=\mu_{x}(x>f(m) \& \lambda(x) \neq 2) \quad(m=0,1,2, \ldots)$.

Also, we extend the functions $\lambda, \varphi$, and $\psi$ by letting

$$
\lambda(-1)=0, \quad \varphi(-1)=0, \quad \psi(-1)=1 .
$$

Using this non-primitive recursive function $f(n)$, we can eliminate $\xi(n)$, $\eta(n), *(n)$, and $\varrho(n)$ from the scheme (4.1). For $m \geqslant 1$ we obtain

$$
\begin{aligned}
& \varphi(f(m+1))=p(f(m)-f(m-1)+2, \psi(f(m)), \psi(f(m-1))) \\
& \varphi(f(m+1))=[\psi(f(m+1)) / \psi(f(m))] \varphi(f(m))+\varphi(f(m-1)) .
\end{aligned}
$$

if $\lambda(f(m)) \neq \lambda(f(m-1))$; and

$\psi(f(m+1))=p(f(m)-f(m-1)+2, \psi(f(m)), \psi(f(m))-\psi(f(m-1)))$,

$\varphi(f(m+1))=[\psi(f(m+1)) \mid \psi(f(m))] \psi(f(m))+\varphi(f(m))-\varphi(f(m-1))$

if $\lambda(f(m))=\lambda(f(m-1))$.

Also we have

$$
\psi(f(1))=2, \quad \varphi(f(1))=1 ;
$$

and if $f(m-1)<n \leqslant f(m)$, then

$$
\psi(n)=\psi(f(m)), \quad \psi(n)=\psi(f(m)) .
$$

From (4.3) it is readily verified by induction that $\psi(f(m))$ is a prime for $m \geqslant 1$, since $\varphi(f(m-1))$ is relatively prime to $w(f(m))$. Hence, by (4.4), $\psi(n)$ is a prime for all $n \geqslant 0$. Also by $(4.3)$ we have for $n \geqslant 1$

$$
\psi(\dot{f}(m+1)) \geqslant(f(m)-f(m-1)+2) \psi(f(m))
$$

Now we prove that the sequence $\{\varphi(n) / \psi(n)\}$ converges to an irrational number by constiucting a continued fraction for which each of the numbers $\varphi(n) / \varphi(n)$ is one of the convergents. Let

$$
g(1)=1, \quad g(m+1)=\left\{\begin{array}{lll}
g(m)+1 & \text { if } \quad \lambda(f(m)) \neq \lambda(f(m-1)) \\
g(m)+2 & \text { if } \quad \lambda(f(m))=\lambda(f(m-1)) .
\end{array}\right.
$$

As usual we set

$$
p_{-1}=1, \quad q_{-1}=0, \quad p_{0}=0, \cdots q_{0}=1, \quad b_{0}=0 ; \cdots:
$$

For brevity, let $k=g(m)$. Then if $\lambda(f(m)) \neq \lambda(f(m-1))$ we let

$(4.6) \quad, \quad q_{k}=\dot{\psi}(f(m)), \quad p_{k}=\varphi(f(m)), \quad b_{k}=\left[q_{k} / q_{k-1}\right]$,

and if $\lambda(f(m))=\lambda(f(m-1))$ we let

$$
\begin{aligned}
& q_{k}=\psi(f(m))-\psi(f(m-1)), \quad p_{k}=\varphi(f(m))-\psi(f(m-1)), \quad b_{k}=\left[q_{k} / q_{k-1}\right], \\
& q_{k+1}=\psi(f(m)), \quad p_{k+1}=\varphi(f(m)), \quad b_{k+1}=1 \text {. }
\end{aligned}
$$

Now let us verify that when $b_{n}, p_{n}$, and $q_{n}$ are defined in this way, the relations (1.1) hold. If $m \geqslant 2$ it follows from $(4.3),(4.6)$ and (4.7) that

$$
\begin{aligned}
& \psi(f(m))=p\left(f(m)-f(m-1)+2, q_{k-1}, q_{k-2}\right), \\
& \varphi(f(m))=\left[\varphi(f(m)) / q_{k-1}\right] p_{k-1}+p_{k-2} .
\end{aligned}
$$


If $\lambda(f(m)) \neq \lambda(f(m-1))$ we obtain

$$
g_{k}=b_{k} q_{k+1}+q_{k-2}, \quad p_{k}=b_{k} p_{k-1}+p_{k-2} ;
$$

and if $\lambda(f(m))=\lambda(f(m-1))$ we obtain

$$
\begin{aligned}
q_{k} & =b_{k} q_{k-1}+q_{k-2}, & p_{k} & =b_{k} p_{k-1}+p_{k-2}, \\
q_{k+1} & =b_{k+1} q_{k}+q_{k-1}, & p_{k-1} & =b_{k+1} p_{k}+p_{k-1} .
\end{aligned}
$$

Thus the recursion relation of (1.1) holds for $n+1 \geqslant g(2)$. One can also verify directly that the recursion relation holds for $n=0$ and 1 and that the other equations of (1.1) hold. We let $a$ be the corresponding irrational number, i. e. $a=\left[0, b_{1}, b_{3}, b_{3}, \ldots\right]$. Observe that each of the numbers $\varphi(n) / \psi(n)(n=0,1,2, \ldots)$ is equal to some convergent.

We shall need an estimate for how closely $\varphi(n) / \psi(n)$ approximates $a$. First we prove by induction that

$$
\psi(f(m+1)) \geqslant f(m) \quad(m=1,2,3, \ldots) .
$$

By (4.5) we have

$$
\psi(f(2)) \geqslant(f(1)+3) \psi(f(1)) \geqslant f(1)
$$

hich proves (4.8) for $m=1$. For $m>1$ we assume the inequality with $m$ replaced by $m-1$ and use (4.5) and the fact that $f(m-1) \geqslant 1$ to get

$$
\begin{aligned}
\psi(f(m+1)) & \geqslant(f(m)-f(m-1)+2) f(m-1) \\
& \geqslant f(m)-f(m-1)+2 f(m-1) \geqslant f(m),
\end{aligned}
$$

thus completing the proof of (4.8).

The fraction $\varphi(f(m)) / \psi(f(m))$ is a convergent to $a$. The next convergent is either $\frac{\varphi(f(m+1))}{\psi(f(m+1))}$ or $\frac{\varphi(f(m+1))-\varphi(f(m))}{\psi(f(m+1))-\psi(f(m))}$. Hence by (1.3) we obtain.

$$
\left|\alpha-\frac{\varphi(f(m))}{\psi(f(m))}\right|<\frac{1}{\psi(f(m))(\psi(f(m+1))-\psi(f(m)))} \leqslant \frac{1}{\psi(f(m+1))} \leqslant \frac{1}{f(m)}
$$

for $m=1,2,3, \ldots$ Consequently, in view of $(4.4)$ we have for all $n \geqslant 1$

$$
\left|a-\frac{\varphi(n)}{\psi(n)}\right|<\frac{1}{n}
$$

Let $b$ be any integer $\geqslant 2$ and suppose

$$
\alpha=\sum_{n=0}^{\infty} \gamma(n) b^{n}
$$

with $0 \leqslant \gamma(n)<b$ for $n=0,1,2, \ldots$ We wish to prove that $\gamma(n)$ is a recursive function. We have

$$
\gamma(0)=[a], \quad \gamma(n+1)=\left[b^{n+1} a\right]-b\left[b^{n} a\right] .
$$

Consequently, it is sufficient to show that $\left[b^{n} a\right]$ is a recursive function of $n$. If possible, let $a$ be an integer for which

$$
\left|\alpha-\frac{a}{b^{n}}\right|<\frac{1}{3 b^{2 n}}
$$

By (1.7), $a / b^{n}$ is equal to some convergent $p_{j} / q_{j}$. Furthermore, by (1.3), $b_{j+1} \geqslant 2$; and, therefore, by (4.6) and (4.7) there is an $m$ such that $p_{j}=\varphi(m)$ $q_{f}=\psi(m)$. Also $a / b^{n}=\varphi(m) / \psi(m)$ is impossible if $\psi(m)>b$ because $\psi(m)$ is a prime. Consequently, there are at most a finite number of $n$ for which there is an $a$ such that (4.10) holds. Let $N$ be the least positive integer which is greater than all such $n$. Then, by (4.9),

$$
\left|b^{n} \frac{\varphi\left(4 b^{2 n}\right)}{\psi\left(4 b^{2 n}\right)}-b^{n} \alpha\right|<\frac{b^{n}}{4 b^{2 n}}=\frac{1}{4 b^{n}} .
$$

Also, if $n \geqslant N$ and $a$ is any integer, then

$$
\left|b^{n} a-a\right| \geqslant \frac{b^{n}}{3 b^{2 n}}=\frac{1}{3 b^{n}} \text {. }
$$

Hence

$$
\begin{array}{lll}
{\left[b^{n} \alpha\right]=\left[b^{n} \varphi\left(4 b^{2 n}\right) / \psi\left(4 b^{2 n}\right)\right]} & \text { if } & n \geqslant N, \\
{\left[b^{n} \alpha\right]=\left[b^{n} \varphi\left(4 b^{2 N}\right) / \psi\left(4 b^{2 N}\right)\right]} & \text { if } & n<N ;
\end{array}
$$

and from this it follows immediately that $\left[b^{n} \alpha\right]$ is a primitive recursive function of $n$.

To complete the proof of Theorem 4, we show that $a$ does not determine a recursive cut, $i$. e. that the relation $m /(n+1)<\alpha$ is not primitive recursive. Using (4.6) and (4.7) one can establish by induction that if $\lambda(f(m))=0$, then $\varphi(f(m)) / \psi(f(m))$ is a convergent of even order; and if $\lambda(f(m))=1$, then $\varphi(f(m)) / \psi(f(m))$ is a convergent of odd order. It then follows from (1.4) and (4.4) that

$$
\frac{\varphi(n)}{\psi(n)}<a \quad \text { if and only if } \quad \lambda\left(\mu_{x}(x \geqslant n \& \lambda(x) \neq 2)=0 .\right.
$$

Hence by Lemma 1 the relation $\varphi(n) / \psi(n)<\alpha$ is not primitive recursive, and thus the relation $m /(n+1)<a$ is also not primitive recursive. 


\section{References}

[1] R. L. Goodstein, The reoursive irrationality of $\pi$, Journ. Symb. Logic 19 (1954), pp. 267-274.

[2] A. Hurwitz, Veber die angenäherte Darstellung der Zahlen durch rationale Britche, Mathematische Annalen 44 (1894), pp. 417-436. Also in Mathematische Werke, vol. 2, Basel 1933, pp. 136-156.

[3] R. S. Lehman, A study of regular contimed fractions, BRL Report No. 1066. Aberdeen Proving Ground 1950.

[4] A. Mostowski, On computable sequences, Fund. Math. 44 (1957), pp. 37-51.

[5] O. Perron, Die Lehre von den Kettenbrüehen, 3rd ed., vol. 1, Stuttgart 1954.

[6] R. Péter, Zum Begriff der rekursiven reellen Zall, Acta Scientiarum Mathematicarnm Szeged 12/A (1950), pp. 239-245.

[7] - Relursive Funfitionen, Budapest 1951.

[8] K. Pracher, Primzahlverteilung, Berlin $195 \%$.

[9] E. Specker, Nicht konstruktiv beweisbare Sätze der Analysis, Journ. Symb Logie 14 (1949), pp. 145-158.

Reģu par la Rédaotion le 18. 11. 1959

\section{On the decomposition of Haar measure in compact groups}

by

\section{R. Ranga Rao and V.S. Varadarajan (Calcutta)}

o. Introduction. The behaviour of singularity under convolution has always been an interesting question. In particular, it may be asked whether the convolution of singular measures is necessarily singular. However, Salem ([5]) has constructed examples of singular measures whose iterates are absolutely continuous. In this paper we examine this question in another direction. The main theorem of this paper asserts that the Haar measure on any infinite compact abelian group can always be written as the convolution of two singular measures. It is also proved that in any non-discrete locally compact abelian group there are singular measures whose convolution is absolutely continuous.

1. Background. Thronghont this paper, with the exception of the last section, we shall be dealing with compact abelian groups. For any locally compact abelian group $G$, we use the symbol $\lambda_{G}$ to denote the Haar measure of $G$. If $G$ is compact, $\lambda_{G}$ is always normalized to have $\lambda_{G}(G)=1$.

If $G$ is any compact abelian group, let $\mathfrak{B}_{0}$ and $\mathfrak{B}$ denote the $\sigma$-field of Baire and Borel subsets respectively. If $G$ is metric $\mathfrak{B}_{0}=\mathfrak{B}$ and in any case $\mathfrak{B}_{0}$ is the smallest $\sigma$-field with respect to which all continuous functions are measurable. The term measure will be used to denote probability measures on $\mathfrak{B}_{0}$. Since every measure on $\mathfrak{B}_{0}$ has a regular unique extension to $\mathfrak{B}$, we may regard the measure as defined on $\mathfrak{B}$ itself and assume its regularity whenever it is necessary. We will have occasion to use the Riesz theorem. This asserts that if $L$ is any non-negative linear functional on $C(G)$ (the space of continuous functions on $G$ ) with $L(1)=1$, then there exists a unique maesure $p$ such that $L(x)=\int_{\sigma} x d p$ for all $x \in C(G)$.

A measure $p$ on a compact group $G$ is called singular if $p([x])=0$ for all $x \in G$ and if there exists a set $A$ such that $p(A)=1$ and $\lambda_{A}(A)=0$. Singularity is a special case of orthogonality. Measures $p$ and $q$ on $G$ are said to be orthogonal if there exists a set $A$ with $p(A)=q(G-A)=0$; in this case we write $p \perp q$. With this notation, $p$ is singular if and only $p([x])=0$ for all $x \in G$ and if $p-\lambda_{G}$. A measure $p$ is said to be absolutely 